\title{
Wittgenstein sobre as provas indutivas
}

\author{
André Porto \\ UFG \\ andre.s.porto@uol.com.br
}

resumo 0 objetivo deste artigo é apresentar uma reconstrução para o tratamento de Wittgenstein para as provas indutivas. Repetidamente o filósofo fala em separar "o que a prova efetivamente demonstra" de algo que ele chama pejorativamente de "prosa". Nossa proposta de solução é ousada: propomos um novo cálculo, que chamamos de "cálculo horizontal", que encarnaria exatamente aquele conteúdo matemático desse tipo de demonstrações de que fala o filósofo.

palavras-chave Wittgenstein; Provas indutivas; infinitude.

\section{Introdução}

Um engano comum para um novato em filosofia da matemática é achar que as várias posições divergentes possam ser organizadas todas em um crescendo de abrangência, com a matemática clássica envolvendo a totalidade dessas propostas. Assim, por exemplo, pensaríamos que, como o intuicionismo é obtido por uma restrição à lógica clássica (à lei do terceiro excluído), qualquer teorema intuicionista seria um teorema clássico. $\mathrm{Na}$ verdade, não é essa a situação "geográfica" das várias abordagens. Por envolverem uma lógica menos exigente, certas teorias matemáticas intuicionistas sequer são consistentes classicamente. Esse é exatamente o caso, por exemplo, da recente análise suave de Kock \& Lawvere (Kock 2006; Bell 1998).

Existe, no entanto, um núcleo matemático comum a virtualmente todas as propostas de reconstrução matemática: a aritmética recursiva primitiRecebido em 14 de março de 2009. Aceito em 24 de agosto de 2009. doispontos, Curitiba, São Carlos, vol. 6, n. 2, p.79-107, outubro, 2009 
va. Se algum método de prova tem lugar cativo no interior de qualquer reformulação da matemática digna desse nome, esse método é certamente o das provas indutivas, coração da aritmética recursiva primitiva. Mesmo as teorias que pretendem alguma restrição a esse tipo de argumento (Nelson 1986; Parikh 1971) aceitam alguma forma de argumento por indução, caso contrário, nada seria demonstrável, salvo as mais básicas trivialidades.

Em seu período médio, Wittgenstein dedica contínua e exaustiva análise exatamente a esse núcleo matemático fundamental, a aritmética recursiva. No entender do filósofo, encontraríamos, já aí, na interpretação tradicional das provas indutivas, a raiz de um imenso engano na compreensão filosófica dessas provas, engano esse que nos levaria diretamente a posições fundacionais completamente inaceitáveis para o filósofo, como a do realismo platônico. Como veremos mais adiante, Wittgenstein tentava insistentemente separar o "conteúdo matemático razoável" da "prosa matemática enganosa em volta dessas provas". No entender do filósofo, haveria, sim, uma maneira adequada, completamente aceitável, de se encarar o conteúdo dessas demonstrações. Porém, isso envolveria uma profunda reorientação da interpretação tradicional desses argumentos matemáticos. E, em seu entender, essa interpretação tradicional estaria envolta em todo tipo de absurdos.

Nenhum outro tema específico de filosofia da matemática mereceu uma atenção tão sustentada e contínua do filósofo quanto o tema das provas indutivas. Encontramos quase sessenta páginas dedicadas exclusivamente a esse tipo de demonstração aritmética nas Observações Filosóficas e no Big Typescript (Wittgesntein 1975, pg 193-205;2005, pg. 443-81). No entanto, a despeito de várias tentativas importantes de análise interpretativa desse material (Shanker, pg 198-215; Stenlung 1990; Frascolla, pg 79-85), os resultados desses esforços parecem desanimadoramente inconclusivos, especialmente dada a centralidade e a imensa importância do assunto para nossa compreensão da matemática.

Como muitas vezes acontece com a exegese daquele filósofo, as dificuldades de entendimento não envolvem tanto a parte negativa de sua proposta, o porquê de sua recusa da interpretação tradicional, mas, sim, sua parte positiva: a circunscrição do que seria, no seu entender, exatamente esse "conteúdo matemático razoável" escondido nessas provas. Nosso objetivo neste artigo é ambicioso: oferecer uma interpretação para a parte 
positiva da proposta de Wittgenstein na forma de uma tradução para outro cálculo. Esse outro cálculo, de natureza algébrica, direta, deveria, de alguma forma, preservar todo o conteúdo da prova indutiva original, indireta. Mas a referência a qualquer objeto abstrato infinito, ou a introdução da noção de "possibilidade em princípio", seria evitada. Assim, teríamos finalmente determinado de forma precisa o que poderia ser o "conteúdo razoável" das provas indutivas pretendido por Wittgenstein.

Desde o início desse nosso projeto de pesquisa, já dispúnhamos de uma idéia de como seria esse "cálculo algébrico" e já dispúnhamos, até mesmo, de umas poucas "traduões" de provas indiretas em provas diretas. Havia, no entanto, um problema. Não conseguíamos "traduzir” a prova da regra conversa à regra II da definição recursiva da soma:

$$
\left\{\begin{array}{l}
0+n=n \\
s x+n=s(x+n)
\end{array}\right.
$$

O principal resultado que vamos apresentar abaixo é uma solução, em nosso entender, completamente satisfatória, para aquela dificuldade.

Como em tantos casos assim, a chegada a essa solução se deu após uma importante mutação na maneira como encarávamos a operação fundamental daquele cálculo, a operação geral determinada pela regra II das definições recursivas das várias funções aritméticas:

$$
f(s n, x)=h(f(n, x))
$$

Antecipando um pouco, abandonamos uma leitura puramente sintática daquela operação em favor de uma abordagem em termos da idéia, proveniente da Teoria das Categorias, da existência de uma função $\boldsymbol{h}$ que faça o seguinte diagrama comutar:

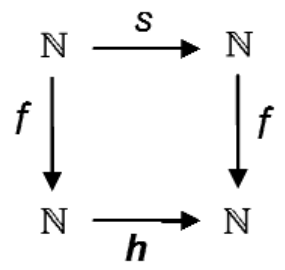


Dividiremos nosso artigo em quatro seções. Na primeira delas, repassaremos rapidamente as raízes para a recusa de Wittgenstein da abordagem tradicional com respeito às provas indutivas. $\mathrm{Na}$ segunda seção, apresentaremos a proposta de "tradução" que havíamos exposto e em seguida, na terceira seção, discutiremos o problema encontrado com a regra conversa da segunda regra recursiva da adição. Por fim, na última seção, apresentaremos a solução que encontramos para aquela dificuldade.

\section{Problemas com a concepção tradicional das provas indutivas}

Talvez o traço mais característico de toda a filosofia da matemática de Wittgenstein seja a sua famosa crítica à idéia de uma regra como "determinando antecipadamente" cada uma de suas aplicações. Assim, em suas palestras de 1939, por exemplo, Wittgenstein afirma:

“Temos a regra de divisão, expressa em termos algébricos ou em termos gerais, - e temos também os exemplos. Sentimo-nos inclinados a dizer, "Mas certamente a regra aponta para a infinitude - voa à sua frente - determina, muito antes de você chegar lá, o que deve fazer." "Determina" - no sentido de que leva você a fazer tal e tal. Mas isso é uma idéia mítica de uma regra - voando através de toda a série aritmética". (Wittgenstein 1976, pg 124) ${ }^{1}$

Vejamos agora uma prova indutiva aritmética ordinária. Em uma prova assim, a partir da demonstração de que uma propriedade qualquer $\boldsymbol{P}$ vale para o zero:

\section{$\vdash P(0)$}

e da demonstração de que, caso essa propriedade valha para um número qualquer $\boldsymbol{n}$, terá de valer também para seu sucessor, $\boldsymbol{s} \boldsymbol{n}$ :

$$
\vdash \forall n(P(n) \rightarrow P(s n))
$$

concluímos que $\boldsymbol{P}$ vale para todos os números naturais 


\section{$\vdash \forall n P(n)$}

Mas, por que nos sentiríamos autorizados a concluir que essa propriedade valha para todos os números baseados apenas na demonstração de que ela vale para o primeiro número, o zero, e de que ela se transfere para o sucessor? A resposta tradicional se dá, mais ou menos, nos seguintes termos. Sabemos que, se $\boldsymbol{P}$ é hereditária, i.e., em todos os casos, ela se transfere para seu sucessor, então em particular sabemos que, caso a propriedade valha para o número 0 , ela valerá também para o número 1 .

$\frac{\forall n(P(n) \rightarrow P(s n))}{P(0) \rightarrow P(1)}$

Mas, como temos também a demonstração de que a propriedade de fato vale para 0 , podemos concluir por modus ponens que:

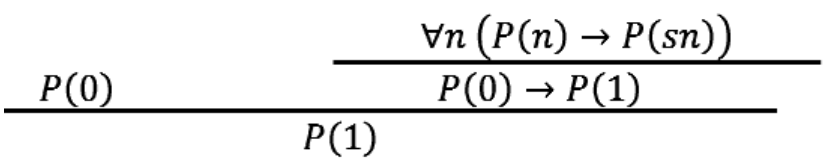

Repetindo os passos acima para os números 1 e 2, poderíamos ir um pouco mais além e concluir também que:

\begin{aligned}$P(0) \quad \frac{\forall n(P(n) \rightarrow P(s n))}{P(0) \rightarrow P(1)} & \\$\cline { 2 - 3 } & $P(1)\end{aligned}$

Teríamos demonstrado então que a propriedade vale para os números 0,1 e 2 . Mas, em que ponto dessa linha argumentativa chegaríamos finalmente a demonstração de que a propriedade $\boldsymbol{P}$ vale para todos os números? É dificil não nos recordarmos aqui das palavras de Wittgenstein na citação acima: temos alguns exemplos e..., de repente,... "a regra aponta para a infinitude - voa à sua frente - determina, muito antes de você chegar lá, o que deve fazer". Claramente, nossa prova somente atingiria seu objetivo, somente demonstraria a propriedade $\boldsymbol{P}$ para todos os números naturais, quando fôssemos 


\section{4}

capazes de ir além dos exemplos (sempre em número finito) e apreendermos a lei geral (infinitária).

Não vamos repassar aqui os vários diferentes aspectos da crítica de Wittgenstein ao tratamento da noção de "infinito". Como deixamos claro na proposta inicial de nossa investigação, a abordagem tradicional com respeito às provas indutivas nos leva, nesse ponto, a duas soluções alternativas igualmente inaceitáveis para o filósofo. A primeira delas, de inspiração clássica, simplesmente introduz um objeto infinitário, a cadeia de inferências:

$$
(\ldots(((P(0) \rightarrow P(1)) \rightarrow P(2)) \rightarrow P(3)) \rightarrow \cdots)
$$

Temos dedicado vários estudos à raiz da crítica de Wittgenstein a essa solução (Porto, 2003; 2007; 2008). Um dos aspectos mais importantes dessa crítica é a recusa da notação por reticências. Essa recusa não se daria em termos epistemológicos, mas, sim, semânticos. Uma notação assim deveria funcionar como um operador que, a partir de um segmento inicial de uma série infinita, produziria um termo singular (que nomearia aquela série). $\mathrm{O}$ problema é que, como sabemos, para qualquer seqüência finita dada, há sempre uma infinidade de regras infinitárias igualmente compatíveis com aquele segmento inicial (finito). Resumidamente, a reclamação do filósofo seria a de que um tal operador não seria aceitável pois não garantiria a unicidade do objeto que pretenderia denotar. Assim, a recusa de Wittgenstein não incidiria inicialmente sobre um tipo de entidade, mas, sim, sobre um tipo de notação.

A segunda solução tradicional para o problema das provas indutivas tem inspiração construtivista. Segundo essa abordagem, deveríamos simplesmente nos abster de introduzir diretamente a existência de um objeto infinitário, aquela seqüencia de inferências. Em seu lugar, deveríamos nos contentar com a noção de existência "potencial" (o infinito só existiria "potencialmente"). Assim, nesse caso, insistiríamos que aquela infinitude de aplicações de modus ponens não "estariam realmente lá", em um mundo platônico, à espera de ser descoberta. Nas palavras de Dummett:

"[...] deveríamos interpor, entre a imagem platonista e a construtivista

[Wittgenstein], uma imagem intermediária, digamos, dos objetos saltando para existência em resposta à nossa exploração (springing into being in response of our probing). Nós não criamos os objetos, mas temos 
de aceitá-los como os encontramos (isto corresponde à prova impondo-se a nós). Mas eles não estavam antecipadamente lá de forma que nossas afirmações sobre eles fossem verdadeiras ou falsas antes que tivéssemos executado as investigações que os trouxeram à existência". (Dummett 1978, pg 447)

Novamente não repassaremos os vários aspectos da crítica de Wittgenstein à noção de "potência" utilizada aqui. Como afirmamos em (Pereira \& Porto 2003, Porto 2007), para que essa solução pudesse funcionar, ela teria de envolver a noção abstrata de "possibilidade em princípio" (e não a idéia de "possibilidade real"). Não estaríamos falando nesse caso em possibilidades que poderiam efetivamente serem levadas a cabo (possibilidades reais). Nossas "possibilidades em princípio" seriam exatamente isso: possibilidades meramente "teóricas". Assim, poderíamos até mesmo falar em processos que envolvessem, para sua execução, digamos, um intervalo de tempo maior do que o número de segundos desde o big bang.

As conseqüências da introdução dessa noção forte de "possibilidade em princípio" nos fundamentos do Intuicionismo são nefastas. Como vários comentadores já apontaram, isso abriria espaço para que os mesmos argumentos que esses intuicionistas se acostumaram a usar para criticar a matemática clássica pudessem, agora, se voltar contra suas próprias propostas:

“[...] argumentos essencialmente análogos àqueles usados pelos intuicionistas matemáticos para justificar sua revisão da lógica clássica e matemática, pelo menos quando sua defesa é apresentada da maneira como Dummett recomenda, levam à uma visão ainda mais radical, a do finitismo estrito; esta visão, no entanto, é incapaz de produzir uma filosofia da matemática coerente; portanto deve haver algo de errado com os argumentos que nos levam à ela e, por analogia, com os argumentos intuicionistas originais também”. (Wright 1993, pg 107)

O problema da elucidação filosófica das provas indutivas continuaria, assim, em aberto. E, como afirmamos no início desse artigo, trata-se de um núcleo central, inegociável para qualquer "filosofia coerente da matemática". Abrir mão das provas indutivas seria abrir mão da aritmética e, conseqüentemente, de qualquer "matemática" reconhecível como tal. O impasse filosófico continuaria. 


\section{6}

\section{A "purificação" das provas indutivas}

Ao longo de todo o seu esforço de esclarecimento filosófico da matemática a partir de 1929, Wittgenstein freqüentemente descreve sua própria atividade de elucidação como visando à separação de uma parte positiva, aquilo que é "realmente demonstrado pela prova", de uma parte negativa, a "prosa matemática enganadora". (Wittgenstein 2005, pg 425-9. A tarefa fundamental do filósofo seria vista então como uma espécie de "purificação" dessa parte positiva, livrando-a da nefasta "prosa matemática".

No caso específico das provas indutivas, o filósofo não parece ter dúvidas em identificar a parte negativa, espúria, dessas demonstrações exatamente com a idéia de uma expansão infinita de aplicações possíveis. Seria, nada mais, nada menos, do que essa idéia, um domínio de infinitos dos números naturais, que deveríamos, de alguma forma, extirpar de nossas provas indutivas!

"Não devemos confundir a possibilidade infinita de aplicação com o que é realmente provado. A possibilidade infinita de aplicação não é provada”! (WITTGENSTEIN, 1975, p. 193)

Mas, como vimos na seção anterior, isso envolveria exatamente abrirmos mão, ou bem do objeto infinito

$$
(\ldots(((P(0) \rightarrow P(1)) \rightarrow P(2)) \rightarrow P(3)) \rightarrow \cdots)
$$

ou bem, pelo menos, da idéia de uma "regra que aponte para o infinito", que "determine potencialmente cada aplicação sua, antecipadamente".

Se seguirmos o conselho de Wittgenstein e olharmos tão somente para o que essas provas "realmente nos dão", a resposta parece ser inescapável: elas nos dão apenas a prova de que uma certa propriedade

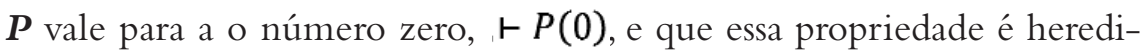
tária, $\vdash \forall n(P(n) \rightarrow P(s n))$. Qualquer conclusão ulterior parece necessariamente envolver o "salto à infinitude": a idéia de que, partindo da base e avançando metodicamente, sucessor a sucessor, acabaríamos por abarcar, ainda que "potencialmente", todos os números. Como poderíamos abrir mão desse componente das provas indutivas sem mutilar completamente seu "conteúdo matemático"? 


\section{0 cálculo equacional de Goodstein}

Wittgenstein abre a seção 127 de seu Big Typescript com uma descrição de qual seria realmente o "conteúdo" da demonstração indutiva de Skolem da propriedade associativa da soma:

O que Skolem chama de uma prova recursiva de A pode ser escrito assim:

$\mathrm{a}+(\mathrm{b}+1)=(\mathrm{a}+\mathrm{b})+1$

$\mathrm{a}+(\mathrm{b}+(\mathrm{c}+1))=\mathrm{a}+((\mathrm{b}+\mathrm{c})+1)=(\mathrm{a}+(\mathrm{b}+\mathrm{c}))+1$

$(a+b)+(c+1)=((a+b)+c)+1$

Nesta prova a proposição provada obviamente não ocorre de torma alguma. - O que temos que fazer é estabelecermos uma estipulação geral permitindo o passo até ela. Esta estipulação poderia ser expressa assim:

$$
\left.\begin{array}{l}
\varphi(1)=\psi(1) \\
\varphi(\mathrm{C}+1)=F(\varphi(\mathrm{C})) \\
\psi(\mathrm{C}+1)=F(\psi(\mathrm{c}))
\end{array}\right\} \quad \begin{gathered}
\Delta \\
\varphi(\mathrm{c})=\psi(\mathrm{c})
\end{gathered}
$$

Se três equações da forma $\alpha, \beta, \gamma$ são provadas, dizemos que "a equação $\Delta$ foi provada para todos os números cardinais". (Wittgenstein 2005, pg 445)

Há vários pontos a serem observados com respeito a essa descrição do que seria o "real conteúdo" daquela prova indutiva, segundo Wittgenstein. Em primeiro lugar, ao invés de dividir a demonstração indireta em duas partes, a prova da base e a prova do passo indutivo, o filósofo prefere dividi-la em três partes, três cadeias equacionais:

1) A prova da base ("equações da base").

2) A parte da prova do passo indutivo até o uso da hipótese indutiva ("equações da esquerda").

3) A parte da prova do passo indutivo depois do uso da hipótese indutiva ("equações da direita").

Em realidade, como atesta a segunda parte do trecho citado de Wittgenstein, essa pequena reorganização dos conteúdos daquela prova esconde de fato uma mudança muito maior na própria compreensão da estrutura abstrata daquela demonstração. 
$\mathrm{Na}$ concepção tradicional, qualquer prova indutiva aritmética envolveria uma série de operações de abstração. Essas operações visariam a obtenção de um predicado, o predicado $[\lambda x . P(x)]$ sobre o qual a prova indutiva se daria. Assim, em nosso exemplo da propriedade associativa da soma, precisaríamos inicialmente transformar uma equação envolvendo três variáveis (de cada lado da equação a ser provada) em uma identidade envolvendo apenas uma variável. Para isso, precisaríamos de duas operações de abstração. Na primeira delas, obteríamos uma identidade sobre duas funções unárias:

$$
\begin{gathered}
(x+(y+n))=((x+y)+n) \\
{[\lambda n \cdot(x+(y+n))](n)=[\lambda n \cdot((x+y)+n)](n)}
\end{gathered}
$$

Após a essa primeira redução por abstração no número de variáveis envolvidas, o passo seguinte seria então, finalmente, a obtenção de um único predicado, o predicado indutivo pretendido, a "propriedade indutiva $\boldsymbol{P}$ " sobre a qual se dará a demonstração:

(3) $[\lambda n \cdot(x+(y+n))=((x+y)+n)](n)=_{\text {def }}[\lambda x \cdot P(x)]$

Todo o maquinário algébrico, equacional teria assim sido ocultado, desaparecido. Seria tão somente sobre esse predicado unário complexo $[\lambda x . P(x)]$ que montaríamos nossa demonstração.

A análise de Wittgenstein reintroduz parte do maquinário equacional, algébrico, como componente essencial da prova. O processo de abstração pararia um passo antes, na abstração (2) acima. Dessa maneira, a forma geral de uma demonstração aritmética indutiva seria montada sobre a identificação de duas funções ordinárias, $\phi$ e $\psi$

$$
\phi(n)=\psi(n)
$$

e não sobre um predicado, $\boldsymbol{P}$.

Além disso, a conclusão final da prova, a demonstração daquela identidade funcional, seria obtida, como diz Wittgenstein, a partir de "uma estipulação geral" (i.e., uma regra inferencial) envolvendo as três formas equacionais, a equação da base: 


$$
\phi(0)=\psi(0)
$$

e as duas equações indutivas (a equação esquerda, até a hipótese, e a direita, depois da hipótese):

$$
\begin{gathered}
\phi(s n)=F(\phi(n)) \\
\psi(s n)=F(\psi(n))
\end{gathered}
$$

O que Wittgenstein chama de "forma geral da estipulação indutiva" na citação acima é exatamente a regra central $\boldsymbol{U}$ do Cálculo Equacional de Reuben Goodstein (GOODSTEIN, 1957, p. 104; MARION, 2008, pp. 101-9).

$$
\begin{gathered}
\phi(0)=\psi(0) \\
\phi(s n)=F(\phi(n)) \\
\psi(s n)=F(\psi(n)) \\
\hline \forall n(\phi(n)=\psi(n))
\end{gathered}
$$

De fato, como esse discípulo de Wittgenstein (seu único orientando) demonstrou em detalhes em (Goodstein 1957), a regra proposta por Wittgenstein é de fato equivalente ao esquema indutivo tradicional:

$$
\frac{P(0) \quad P(n) \rightarrow P(s n)}{\forall n P(n)}
$$

Voltemos para a regra $\boldsymbol{U}$ de Wittgenstein/Goodstein. Expressas na linguagem da moderna Teoria das Categorias, cada uma das duas equações indutivas (as equações da esquerda, e da direita) determinaria a existência de uma função $\boldsymbol{F}$ que faria ambos os diagramas comutarem:

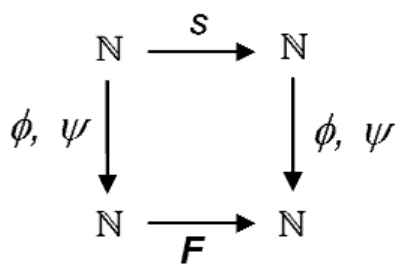


Nem tudo estaria resolvido, no entanto, apenas com a introdução dessa nova regra $\boldsymbol{U}$, em substituição ao esquema indutivo tradicional. No cálculo de Goodstein, reencontramos a nefasta referência à "possibilidade infinita de aplicação", proscrita por Wittgenstein. Novamente, se perguntarmos pela justificação da conclusão da nova regra, $\forall n(\phi(n)=\psi(n))$ receberíamos como resposta algo como a explicação abaixo.

Pela equação da base, sabemos da identidade dos valores das funções $\phi$.e $\psi$ para o número 0

$$
\phi(0)=\psi(0)
$$

Mas, pela regra de que a aplicação de uma mesma função a argumentos idênticos produz resultados idênticos

$$
\frac{a=b}{f(a)=f(b)}
$$

para o caso específico da identidade $\phi(0)=\psi(0)$ e daquela função $\boldsymbol{F}$ (a função obtida pela comutação), poderíamos concluir então que

$$
\frac{\phi(0)=\psi(0)}{F(\phi(0))=F(\psi(0))}
$$

Mas, como a função $\boldsymbol{F}$ comuta com as funções $\phi$ e $\psi$, poderemos "internalizar", de cada lado daquela equação, aquela tunção $\boldsymbol{F}$ em termos da função sucessor.

$$
\frac{\phi(0)=\psi(0)}{\phi(s(0))=F(\phi(0))=F(\psi(0))=\psi(s(0))}
$$

O resultado final de nosso argumento é que, por transitividade da identidade, obtemos

$$
\frac{\phi(0)=\psi(0)}{\phi(s(0))=\psi(s(0))}
$$


ou seja, o valor das duas funções é o mesmo também para o número

$$
\phi(1)=\psi(1)
$$

Repetindo a mesma argumentação para a identidade $\phi(1)=\psi(1)$, obteríamos

$$
\frac{\phi(0)=\psi(0)}{\frac{\phi(s(0))=F(\phi(0))=F(\psi(0))=\psi(s(0))}{\phi(s s(0))=F(\phi(s(0)))=\quad=F(\psi(s(0)))=\psi(\operatorname{ss}(0))}}
$$

ou seja, obteríamos que

$$
\phi(2)=\psi(2)
$$

A essa altura de nossa argumentação nos encontraríamos novamente frente à pergunta que nos levava à operação proscrita do "pulo ao infinito": "em que ponto dessa linha argumentativa chegaríamos finalmente a demonstração de que a identidade entre essas duas funções vale para todos os números? Novamente, gostaríamos de dizer que, se temos que

$$
\phi(0)=\psi(0)
$$

e temos um argumento para passar de $\phi(0)=\psi(0)$ para $\phi(s n)=\psi(s n)$

$$
\phi(n)=\psi(n) \rightarrow \phi(s n)=\psi(s n)
$$

então poderíamos seguir o caminho apontado pelas reticências...

$$
\begin{gathered}
\frac{\phi(0)=\psi(0)}{\phi(s(0))=F(\phi(0))=F(\psi(0))=\psi(s(0))} \\
\hline \phi(s s(0))=F(\phi(s(0)))=\quad \\
\hline
\end{gathered}
$$


e concluirmos que demonstramos aquela identidade para todos os números naturais.

Mas, novamente aqui, é claro, estaríamos incluindo, como parte do conteúdo de nossa demonstração, o "salto ao infinito" rejeitado por Wittgenstein. Estaríamos nos comprometendo com a idéia de que, para qualquer número natural, mesmo um número imenso como

$$
10^{10^{10^{10}}}
$$

"poderíamos em princípio obter", nada mais, nada menos, do que uma demonstração daquela imensa cadeia de inferências, determinando "finalmente" que:

$$
\phi\left(10^{10^{10^{10}}}\right)=\psi\left(10^{10^{10^{10}}}\right)
$$

Essa "capacidade", no entanto, teria de ser tomada como sendo "meramente teórica". Teríamos distinguido, assim, dois tipos de possibilidades, uma real e outra "teórica". Em termos de possibilidades reais, claramente não existiria a possibilidade de demonstrarmos a equação (5) partindo da equação $\phi(0)=\psi(0)$ e seguindo, sucessor a sucessor, cada passo argumentativo como aqueles apresentados em (4). Essa possibilidade não existiria, no entanto, apenas no "plano real". Em um "plano teórico" essa possibilidade "estaria lá". Ela seria semelhante a casas decimais ainda não calculadas de Pi.A seqüencia inteira (de decimais de $\mathrm{Pi}$, ou de passos argumentativos de nossa prova) estaria à espera de que alguém a "atualize no plano real", já que sua existência já estaria "potencialmente dada" em um "plano teórico".

Multiplicamos 25 x 25 e obtemos 625. Mas no mundo matemático 25 x 25 já são 625.

A idéia é que existe um reino da geometria em que as entidades geométricas existem. O que no mundo ordinário chamaríamos de possibilidade no mundo geométrico seria realidade. (Wittgenstein 1976,pg 145, 144)

\section{A abordagem horizontal de Wittgenstein e a operação de iteração}


Voltemos à figura (4) acima.

$$
\frac{\frac{\phi(0)=\psi(0)}{\phi(s(0))=F(\phi(0))=F(\psi(0))=\psi(s(0))}}{\phi(s s(0))=F(\phi(s(0)))=\quad=F(\psi(s(0)))=\psi(s s(0))}
$$

Nossa sugestão é de que, ao invés da abordagem vertical de Goodstein (que partiria de uma equação da base a ascenderia verticalmente, passo a passo, ao infinito), Wittgenstein propunha uma outra abordagem, uma abordagem horizontal.

Comecemos do lado esquerdo daquela estrutura:

$$
\phi(s s(0))
$$

Comutando um sucessor pela função $\boldsymbol{F}$ obteríamos:

$$
\phi(s s(0))=\boldsymbol{F}(\boldsymbol{\phi}(\boldsymbol{s}(\mathbf{0})))
$$

Aplicando mais uma vez a operação de comutação, obteríamos:

$$
\phi(s s(0))=F(\phi(s(0)))=\boldsymbol{F}(\boldsymbol{F}(\boldsymbol{\phi}(\mathbf{0})))
$$

Mas, como temos a identidade da base, $\phi(0)=\psi(0)$ podemos avançar (horizontalmente) mais um passo e obter

$$
\phi(s s(0))=F(\phi(s(0)))=F(F(\phi(0)))=\boldsymbol{F}(\boldsymbol{F}(\boldsymbol{\psi}(\mathbf{0})))
$$

Agora, só nos restaria "internalizar", uma por uma, as ocorrências da função $F$

$$
\phi(s s(0))=F(\phi(s(0)))=F(F(\phi(0)))=F(F(\psi(0)))=\boldsymbol{F}(\boldsymbol{\psi}(\mathbf{s} \mathbf{0}))
$$

e finalmente

$$
\phi(s s(0))=F(\phi(s(0)))=F(F(\phi(0)))=F(F(\psi(0)))=F(\psi(s 0))=\boldsymbol{\psi}(\boldsymbol{s s} \mathbf{0})
$$


de forma a obtermos, horizontalmente, uma prova de que:

$$
\phi(s s(0))=\psi(s s 0)
$$

Obtivemos o mesmo resultado, a mesma identidade, do da figura (4) acima, mas, ao invés de seguirmos um movimento argumentativo vertical, ao longo de inferências representadas pelas barras horizontais

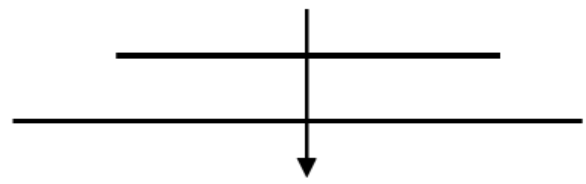

fizemos um movimento horizontal, puramente algébrico, i.e., obtido, não por inferências, mas, sim, por substituições e pela transitividade da identidade.

$$
\phi(s s(0))=\quad \longrightarrow \quad=\psi(s s 0)
$$

Logramos demonstrar a mesma equação

$$
\phi(2)=\psi(2)
$$

por um método paralelo, horizontal e algébrico, sem termos de percorrer à linha argumentativa vertical, de Goodstein. No entanto, é importante termos em mente nosso objetivo: evitar o recurso às reticências. Como vimos, pelo método vertical (da mesma forma que no caso das provas indutivas ordinárias), para atingirmos o caso geral

$$
\phi(n)=\psi(n)
$$

precisaríamos recorrer ao famigerado recurso à operação "salto ao infinito", às reticências.

Porém, o novo método horizontal ainda não foi capaz de demonstrar a equação geral (6). Tudo o que obtivemos até agora foi a aplicação do novo método ao caso particular de uma identidade específica, aquela singela identidade apresentada na equação (5). Como deveríamos proceder para apresentar uma prova horizontal da equação geral (6) pelo novo método horizontal? 
A resposta está em uma antiga idéia do Tractatus de Wittgenstein. No sistema aritmético apresentado naquele livro 2 , o filósofo substitui as usuais definições, através de esquemas recursivos de cada uma das operações aritméticas usuais como a adição e a multiplicação,

$$
\left\{\begin{array} { l } 
{ ( x + 0 ) = x } \\
{ ( x + s y ) = s ( x + y ) }
\end{array} \quad \left\{\begin{array}{l}
(x \times 0)=0 \\
(x \times s y)=((x \times y)+x)
\end{array}\right.\right.
$$

por uma única definição geral de iteração de uma operação qualquer $\Omega$ (sobre uma base qualquer $\boldsymbol{b}$ )

$$
\begin{cases}(\Omega)^{0} b & b \\ (\Omega)^{s n} b & \Omega\left((\Omega)^{n} b\right)\end{cases}
$$

De posse dessa definição geral de "iteração", o Tractatus oferece então definições diretas para todas aquelas operações aritméticas que antes seriam definidas indiretamente, por recursão. Por exemplo, no caso da adição e da multiplicação, temos as definições diretas (Wittgenstein 1961, 6.241)

$$
\begin{aligned}
& \text { Adição } \\
& n+m==_{\text {def }}[\Omega]^{n}\left([\Omega]^{m} 0\right) \quad n \times m==_{\text {def }}\left([\Omega]^{n}\right)^{m} 0
\end{aligned}
$$

a operação de adição seria apenas a operação de composição de funções e a operação de multiplicação seria a operação de iteração de adições.

Voltemos agora, uma vez mais à figura (4) acima. Usando a notação de iteração funcional, poderíamos oferecer uma "versão geral" daquela figura, mais ou menos como:

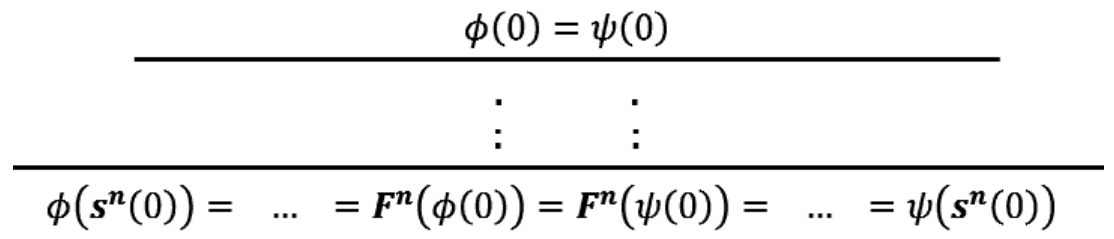

Essa figura apresenta a estrutura geral de uma prova de Goodstein para um número qualquer $\boldsymbol{n}$. 
Mas podemos ir mais além. Se agora generalizássemos as equações esquerda e direita da regra $\boldsymbol{U}$ de Wittgenstein/Goodstein

$$
\phi(s n)=F(\phi(n)) \quad \text { e } \quad \psi(s n)=F(\psi(n))
$$

iterativamente como

$$
\phi\left(s^{n} 0\right)=F^{n}(\phi(0)) \quad \text { e } \quad \psi\left(s^{n} 0\right)=F^{n}(\psi(0))
$$

poderíamos finalmente obter a "tradução vertical" de toda aquela prova, simplesmente como:

$$
\phi\left(\boldsymbol{s}^{n} 0\right)=\boldsymbol{F}^{n}(\phi(0))=\boldsymbol{F}^{n}(\psi(0))=\psi\left(\boldsymbol{s}^{n} 0\right)
$$

Essa seqüência de equações exprimiria exatamente a estrutura abstrata geral envolvida em uma prova indutiva (qualquer) para o caso geral de um número $\boldsymbol{n}$ :

(1) $\boldsymbol{n}$ comutações da operação sucessor pela função $\boldsymbol{F}$, as "externalizações":

$\phi\left(\boldsymbol{s}^{\boldsymbol{n}} 0\right)=\boldsymbol{F}^{\boldsymbol{n}}(\phi(0))$

(2) A substituição funcional na base, $\boldsymbol{F}^{\boldsymbol{n}}(\phi(0))=\boldsymbol{F}^{\boldsymbol{n}}(\psi(0))$

(3) $\boldsymbol{n}$ comutações da função $\boldsymbol{F}$ pela operação sucessor, as "internalizações":

$$
\boldsymbol{F}^{\boldsymbol{n}}(\psi(0))=\psi\left(\boldsymbol{s}^{\boldsymbol{n}} 0\right)
$$

Mas, a passagem da aplicação do argumento a um número específico para o caso geral teria sido obtida recorrendo-se, não a introdução de reticências à direita, i.e., reticências ilimitadas (como no caso de Goodstein):

$$
((\phi(n)=\psi(n) \rightarrow \phi(s n)=\psi(s n)) \rightarrow \phi(s n)=\psi(s n))
$$

mas, sim, apelando-se, alternativamente, a reticências intermediárias, i.e., reticências cotadas:

$$
\phi\left(s^{n}(0)\right)=\quad \ldots \quad=F^{n}(\phi(0))=F^{n}(\psi(0))=\quad \ldots \quad=\psi\left(s^{n}(0)\right)
$$


Alternativamente, como vimos, poderíamos exprimir a mesma idéia através de uma única operação de comutação iterada, $C^{n}$. Escreveríamos, simplesmente:

\section{$\boldsymbol{C}^{\boldsymbol{n}}\left[\boldsymbol{\phi}\left(\boldsymbol{s}^{\boldsymbol{n}} \mathbf{0}\right)\right]=F^{n}(\phi(0))=F^{n}(\psi(0))=\boldsymbol{C}^{n}\left[\boldsymbol{\psi}\left(\boldsymbol{s}^{n} \mathbf{0}\right)\right]$}

\section{Tradução horizontal de provas indutivas ordinárias}

Vejamos como ficam essas idéias mais gerais quando aplicadas a provas indutivas específicas. Como sabemos, a própria estrutura de uma prova assim depende das definições recursivas subjacentes às operações aritméticas envolvidas. Tomemos então uma prova indutiva bem simples, como a demonstração da propriedade associativa da adição. No caso da demonstração dessa propriedade, a única definição recursiva envolvida seria a própria definição recursiva da Adição ${ }^{4}$ :

$$
\begin{array}{ll}
\text { i) } \quad(x+0)=x \\
\text { ii) } \quad(x+s n)=s(x+n)
\end{array}
$$

Consideremos agora a segunda regra (ii) dessa definição. Essa regra nos permite "externalizar", para fora do parênteses, uma incidência da operação sucessor

$$
(x+s n)=s(x+n)
$$

Como sabemos, em um cálculo de uma soma específica, como por exemplo $2+3$, precisamos recorrer a repetidas aplicações dessa regra até que a variável indutiva seja "zerada”, quando então aplicamos a regra da base, (i) e obtemos o resultado final:

$$
\begin{array}{cc}
s s 0+s s s 0 & \text { (ii) } \\
s(s s 0+s s 0) & \text { (ii) } \\
s s(s s 0+s 0) & \text { (ii) } \\
s s s(s s 0+0) & \text { (i) } \\
s s s s 0 &
\end{array}
$$


Introduziremos agora uma pequena alteração da regra (ii) da adição. Usando nossa notação para iteração de operações, substituiremos aquela regra por uma nova versão mais geral, iterada, que nos permite "externalizar", de uma vez só, $\boldsymbol{n}$ ocorrências da operação de sucessão.

(ii')

$$
\left(x+s^{n} m\right)=s^{n}(x+m)
$$

Com base naquela regra, podemos reescrever o cálculo acima como envolvendo apenas duas operações: a aplicação da operação de externalização de $\boldsymbol{n}$ sucessores e uma aplicação da regra da base:

$$
\begin{gathered}
s s 0+s s s 0 \\
s s s(s s 0+0) \\
s s s s s 0
\end{gathered}
$$

Em nosso novo cálculo, usaremos, não a regra (ii) ordinária, que nos permite apenas uma comutação de cada vez, mas, sim, a regra iterada (ii'), que generaliza essa permissão para o caso de $\boldsymbol{n}$ aplicações. Mantermos inalterada a mesma regra original para a base. Nossa nova definição da operação de Adição será então:

$$
\begin{aligned}
& \text { i) } \quad x+0=x \\
& \text { ii') } \quad x+s^{n} m=s^{n}(x+m)
\end{aligned}
$$

Consideremos agora a demonstração da propriedade associativa da adição. A prova indutiva ordinária daquela propriedade é mais ou menos a seguinte:

\begin{tabular}{|c|c|}
\hline Base: & Associatividade da adição \\
1) & $a+(b+0)=a+b$ \\
2) & $a+b=(a+b)+0$ \\
Passo indutivo: & \\
1) $\quad a+(b+s(n))=a+s(b+n)$ \\
2) & $a+s(b+n)=s(a+(b+n))$ \\
3) & $\boldsymbol{s}(\boldsymbol{a}+(\boldsymbol{b}+\boldsymbol{n}))=\boldsymbol{s}((\boldsymbol{a}+\boldsymbol{b})+\boldsymbol{n})$ \\
4) & $s((a+b)+n)=(a+b)+s(n)$ \\
\hline
\end{tabular}


Começaremos nossa "tradução" daquela prova indutiva em uma prova direta, algébrica, considerando a seqüencia esquerda de equações (as equações (1) e (2) no interior da demonstração do "passo indutivo", até o uso da hipótese indutiva):

$$
a+(b+\boldsymbol{s}(n))=a+\boldsymbol{s}(b+n)=\boldsymbol{s}(a+(b+n))
$$

Essa seqüência nos apresenta uma serie de externalizações. Recorrendo agora à notação iterativa e a nossa nova regra (ii'), podemos exprimir simultaneamente $\boldsymbol{n}$ ocorrências dessa operação. Obteríamos então a seguinte "tradução" para aquela seqüência de equações

$$
a+\left(b+s^{n}(0)\right)=a+s^{n}(b+0)=s^{n}(a+(b+0))
$$

Com a operação sucessor "completamente externalizada", podemos agora seguir as transformações indicadas pelas equações da base da prova original, e obter as equações:

$$
\boldsymbol{s}^{n}(a+(b+0))=\boldsymbol{s}^{n}(a+b)=\boldsymbol{s}^{n}((a+b)+0)
$$

Retornando para a operação representada pela equação direita do passo indutivo (na prova original, a equação (4), depois da hipótese indutiva), internalizamos novamente a operação sucessor, obtendo finalmente:

$$
\boldsymbol{s}^{n}((a+b)+0)=(a+b)+s^{n}(0)
$$

Se juntarmos todas essas equações em uma única seqüência, obtemos, nada mais, nada menos, do que uma prova direta, algébrica, da propriedade associativa da Adição:

$$
\begin{array}{lr}
a+(b+n) \\
\| \\
a+\left(b+s^{n}(0)\right)=a+s^{n}(b+0)=s^{n}(a+(b+0))=s^{n}(a+b)=s^{n}((a+b)+0)=(a+b)+s^{n}(0)
\end{array}
$$

Como queria Wittgenstein, essa prova preserva de certa forma o "conteúdo matemático" da prova indutiva original. Mas a demonstração original, indutiva, envolvia necessariamente três passos lógicos 
(1) Demonstração da base

(2) Demonstração do passo indutivo

(3) "Salto ao infinito"

incluindo o passo (3), o "salto ao infinito", que estendia os resultados obtidos nos dois primeiros passos (1) e (2) ao longo de toda a seqüência dos números naturais. Já a nova prova horizontal substitui o apelo à idéia (3) (rejeitada por Wittgenstein) por uma aplicação iterada da regra (ii) da Adição, uma generalização muito natural daquela regra.

Temos agora apenas dois tipos de regras, a regra original da base, e a nova regra comutadora generalizada, iterada. A nova demonstração direta não "mistura a estrutura do objeto infinito $0,1,2,3, \ldots$,.. (i.e, o domínio dos números naturais) com a própria estrutura da prova (a seqüência de equações algébricas gerais). Segregamos claramente o conteúdo da prova original em dois planos. Temos um primeiro plano das operações algébricas, gerais, que constituem a nova demonstração. Mas a referência a aplicações especificas dessas regras a números naturais foi simplesmente extirpada da nova prova e relegada a um segundo plano, o plano da aplicação dessas regras. Como queria Wittgenstein, "a possibilidade infinita de aplicação não é provada", não faz parte da nova demonstração. Separamos assim o "conteúdo matemático positivo" da prova original daquela estranha "prosa matemática" a respeito de "possibilidades meramente teóricas", i.e. de "possibilidades impossíveis" (de serem instanciadas).

Ainda podemos, é claro, aplicar a estratégia algébrica apresentada naquela demonstração geral a números específicos, como, por exemplo 5, 4 e 3

$$
\begin{gathered}
5+(4+3) \\
\| \\
5+\left(4+s^{3}(0)\right)=5+s^{3}(4+0)=s^{3}(5+(4+0))=s^{3}(5+4)=s^{3}((5+4)+0)=\left(\begin{array}{c}
(5+4)+3 \\
\|
\end{array}\right.
\end{gathered}
$$

Mas essa possibilidade de aplicação a um caso particular não faz parte da estrutura argumentativa da nova prova "horizontal". Nas palavras de Wittgenstein: “A possibilidade infinita de aplicação não é provada!”. O conteúdo algébrico e a aplicação a números específicos estão claramente distinguidos na nova abordagem horizontal.

Nos interessa aqui, também, aquele exemplo específico de aplicação da nova demonstração horizontal da associatividade da soma (aos números 5, 
4 e 3) porque encontramos, no Big Typescript de Wittgenstein, exatamente essa mesma seqüência horizontal de operações ${ }^{4}$ :

$$
\begin{aligned}
& 5+(4+3)=5+(4+(2+1))=5+((4+2)+1)=(5+(4+2))+1=(5+(4+(1+1)))+ \\
& 1=(5+((4+1)+1))+1=((5+(4+1))+1)+1=(((5+4)+1)+1)+1=(((5+4)+2) \\
& +1=(((5+4)+3)(\text { Wittgenstein 2005, pg 468) }
\end{aligned}
$$

\section{A tradução da conversa da segunda regra da adição}

O processo de tradução das provas indutivas originais em provas iteradas diretas é, em geral, completamente imediato. Vamos traduzindo para o novo cálculo iterado as equações da direita, da base e da esquerda da demonstração original e... obtemos uma prova direta da equação pretendida. Há, no entanto, um caso interessante, o caso da equação conversa da segunda regra da definição recursiva da Adição:

$$
s a+n=s(a+n)
$$

A demonstração indutiva ordinária daquela equação é

\begin{tabular}{|cl|}
\hline Base: & \multicolumn{1}{c|}{$\boldsymbol{s}+\boldsymbol{n}=\mathbf{s}(\boldsymbol{a}+\boldsymbol{n})$} \\
1 & $s a+0=s a$ \\
$2 \quad s a=s(a+0)$ \\
Passo indutivo: \\
$1 \quad s a+s n=s(s a+n)$ \\
$2 \quad s(s a+n)=s s(a+n)$ \\
$3 \quad s s(a+n)=s(a+s n)$ \\
\hline
\end{tabular}

Traduzindo para os termos de nosso novo cálculo, a equação equivalente àquela equação conversa seria:

$$
s^{m} \mathbf{0}+s^{n} \mathbf{0}=s^{m}\left(0+s^{n} 0\right)
$$

Assim, uma demonstração daquela equação no novo cálculo seria uma seqüência de equações que, partindo do termo esquerdo daquela identidade, chegasse até o direito usando apenas as regras (i) e (ii') do novo 
cálculo. Mas, quando vamos aplicar nossa estratégia de tradução, vertendo as equações da esquerda, da direita e da base (da prova original) para o novo cálculo, percebemos que aquelas seqüências equacionais não parecem se "conectar", "não se encontram".

Vejamos o que acontece, em detalhes. Começamos, como sempre, na equação da esquerda (a equação (1), no interior do passo indutivo, até o uso da hipótese). Ela nos sugere apenas uma simples externalização:

$$
s^{m} 0+s^{n} 0=s^{n}\left(s^{m} 0+0\right)
$$

Se juntarmos agora também as equações da base, obteremos a seqüência:

$$
s^{m} 0+s^{n} 0=s^{n}\left(s^{m} 0+0\right)=s^{n} s^{m} 0
$$

Mas, partindo da equação da direita, obtemos

$$
s^{m}\left(0+s^{n} 0\right)=s^{m} s^{n}(0+0)=s^{m} s^{n} 0
$$

As duas cadeias equacionais não se encontram no meio! As seqüências de sucessores que obtemos, vindo do lado esquerdo, e do direito, não são as mesmas!

(D) $s^{m} 0+s^{n} 0=s^{n}\left(s^{m} 0+0\right)=\begin{aligned} & s^{n} s^{m} 0 \\ & s^{m} s^{n} 0=s^{m} s^{n}(0+0)=s^{m}\left(0+s^{n} 0\right)\end{aligned}$

Claro, se pudéssemos comutar as aplicações de sucessores

$$
s^{n} s^{m} \mathbf{0}
$$

para

$$
\boldsymbol{s}^{\boldsymbol{m}} \boldsymbol{s}^{n} \mathbf{0}
$$

teríamos demonstrado a regra conversa. Mas há claramente dois problemas aqui.

1) $O$ que nos autorizaria a fazer tal comutação? Essa operação faria parte do nosso cálculo?

2) Antes mesmo de nos perguntarmos por uma solução assim, precisaríamos saber, de início, porque esse problema apareceu em nossa tradução? Há alguma coisa errada com nosso cálculo? 
Começaremos por tentar elucidar a segunda questão. Há um ponto importante a ser observado. Se considerarmos as duas cadeias equacionais da prova original, observaremos algo curioso: elas efetivamente "se encontram no meio", em uma mesma expressão "ss $(\boldsymbol{a}+\boldsymbol{n})$ ":

$$
s a+s n=s(s a+n)=\begin{aligned}
& s s(a+n) \\
& s s(a+n)=s(a+s n)
\end{aligned}
$$

A razão é simples. Se identificarmos dentro do cálculo, a origem dos vários sucessores (em negrito, na figura acima), perceberemos que o desencontro permanece:

$$
\begin{aligned}
& s a+\boldsymbol{S} n=\boldsymbol{S}(s a+n)= \\
& \boldsymbol{S} s(a+n) \\
& s \boldsymbol{S}(a+n)=s(a+\boldsymbol{S} n)
\end{aligned}
$$

Do ponto de vista da origem da seqüência de sucessores, as duas duplas são, de fato, diferentes. Mas, como o que conta nas provas indutivas é a identidade sintática entre as duplas (e não a identidade de origens), a conexão se dá.

Nosso problema aparece porque, diferentemente de nossa nova estratégia horizontal de prova, a abordagem tradicional envolve a apenas um único passo indutivo. Ao invés de considerarmos as conexões entre $\boldsymbol{n}$ substituições e comutações, restringimos nossa atenção a apenas "uma camada" de execuções daquela operação. Toda a generalização para o caso de $\boldsymbol{n}$ aplicações é deixada para a cadeia inferencial infinita

$$
(\ldots(((P(0) \rightarrow P(1)) \rightarrow P(2)) \rightarrow P(3)) \rightarrow \cdots)
$$

Daí nossa dificuldade: para demonstrar a equação em nosso novo cálculo, precisaríamos de mais um passo, mais uma comutação, passo esse que fica oculto na prova indutiva original, mas que é claramente necessário no novo cálculo horizontal.

Sabemos a raiz de nossa dificuldade: a segunda questão que nos propusemos acima. Nosso próximo desafio é agora a primeira dificuldade arrolada. Qual a justificativa para introduzirmos a identidade 


$$
s^{n} s^{m} 0 \stackrel{?}{=} s^{m} s^{n} 0
$$

requerida para estabelecer a conexão entre as seqüências direita e esquerda e assim completar a prova exigida?

$\mathrm{Na}$ verdade, a raiz de nossa dificuldade está em como interpretar uma expressão iterada como

$$
s^{m} s^{n} 0
$$

em termos de operações binárias. Precisamos de uma regra de tradução de operações unárias em operações binárias, (e vice-versa). ${ }^{5}$

A solução natural, é claro, seria adotarmos a regra:

$$
s^{x}\left(s^{y}(0)\right)=x+y
$$

De fato, adotando essa regra, poderíamos substituir a regra (i) da definição recursiva original por

$$
s^{0}(x)=\mathbb{I}(x)=x
$$

A antiga regra (i) seria então apenas o caso especial:

$$
x+0=s^{x}\left(s^{0}(0)\right)=s^{x}(\mathbb{I}(0))=s^{x}(0)=x
$$

Voltando agora para o caso da identidade que nos permitira completar a demonstração da regra conversa (ii) da Adição:

$$
s^{n} s^{m} 0 \stackrel{?}{=} s^{m} s^{n} 0
$$

Partindo do termo esquerdo daquela identidade e usando a definição (D1) obteríamos:

$$
s^{n} s^{m} 0=s^{n} 0+s^{m} 0
$$

Podemos agora aplicar a regra (ii') da definição de adição e obter 


$$
s^{n} 0+s^{m} 0=s^{m}\left(s^{n} 0+0\right)
$$

Finalmente, aplicando a regra (i) da "definição" de adição (ou, alternativamente, as regras (D1) e (D2)), obtemos a conexão pretendida

$$
s^{m}\left(s^{n} 0+0\right)=s^{m}\left(s^{n} 0\right)
$$

A demonstração completa da equação conversa da segunda regra da definição recursiva da Adição seria então a seqüência:

$\boldsymbol{s}^{\boldsymbol{m}} \mathbf{0}+\boldsymbol{s}^{\boldsymbol{n}} \mathbf{0}=s^{n}\left(s^{m} 0+0\right)=\boldsymbol{s}^{n} \boldsymbol{s}^{m} \mathbf{0}=\boldsymbol{s}^{n} \mathbf{0}+\boldsymbol{s}^{m} \mathbf{0}=\boldsymbol{s}^{\boldsymbol{m}}\left(\boldsymbol{s}^{n} \mathbf{0}+\mathbf{0}\right)=\boldsymbol{s}^{\boldsymbol{m}}\left(\boldsymbol{s}^{n} \mathbf{0}\right)$

$=s^{m} s^{n}(0+0)=\boldsymbol{s}^{\boldsymbol{m}}\left(\mathbf{0}+\boldsymbol{s}^{\boldsymbol{n}} \mathbf{0}\right)$

Um último comentário, antes de encerrarmos, com respeito à interpretação que o filósofo adotaria para essas operações e identidades que vimos discutindo. Como apresentamos em detalhes em (Porto 2008; 2009), em seu período final Wittgenstein adota uma nova concepção de identidades matemáticas em termos da idéia de "correção cruzada". No linguajar do filósofo, estaríamos adotando "novos critérios" (metalingüísticos) para "julgar execuções empíricas" daquelas operações (aritméticas) envolvendo números específicos.

${ }^{1}$ Ver também a famosa (Wittgenstein 2005a, pg 64, \$188)

2 (Wittgenstein 19616.02 em diante).Ver também (Frascolla 1994; 1997)

3 Temos aqui uma opção inicial a ser feita: qual das duas parcelas da adição receberá a variável indutiva. Nossa escolha aqui incidiu sobre a segunda parcela.

${ }^{4}$ A despeito, é claro, da notação distinta para a operação sucessor, “x + 1", e da escolha do 1, e não do 0 , como a base do sistema

5 Essa é uma operação lógica fundamental na teoria das categorias, é claro, para obtermos uma categoria cartesiana fechada. 


\section{Referências bibliograficas}

BELL, J. (1998). A Primer of Infinitesimal Analysis. Cambridge:

Cambridge University Press.

DUMMETT, M. (1978). Truth and other enigmas. Cambridge: Harvard Press.

FRASCOLLA, P. (1997). The Tractatus System of Arithmetic. Synthese, 112, pp. 353-378. (1994). Wittgenstein's Philosophy of Mathematics. Londres:

Routledge.

GOODSTEIN, R. (1957). Recursive Number Theory. Amsterdam: North-Holand Publishing Company.

KOCK, A. (2006). Sythetic Differential Geometry. Cambridge: Cambridge University Press.

MARION, M. (2008). Wittgenstein, Finitism and the Foundations of Mathematics. Oxford: Oxford University Press.

NELSON, E. (1986). Predicative Arithemetic. Princeton University Press: Princeton.

PARIKH, R. (1971). Existence and Feasibility in Arithmetic. The Journal of Symbolic Logic, 36, pp. 494-508.

PEREIRA, L. C., \& PORTO, A. (2003). Algumas considerações sobre a Noção Construtiva de Verdade. O que nos faz pensar, 17, pp. 107-123.

PORTO, A. (2003). As Dízimas Periódicas na Filosofia da Matemática de Wittgenstein. Philósophos, 8, pp. 127-157.

. (2007). Formalization and Infinity. In: W. CARNIELI, \&

J. DA SILVA, Logic, Language and Knowledge: Essays on Chateuabriand's Logical Forms (Vol. 30). Unicamp.

. (2009). Wittgenstein on Mathematical Identities. (em processo de publicação).

. (2008). Wittgenstein, Dízimas periódias e Correção 
Cruazada. CLE e-prints (on line), 8, p. 29.

SHANKER, S. (1987). Wittgenstein and the turning point in the Philosophy of Mathematics. Londres: Croom Helm.

STENLUND, S. (1990). Language and Philosophical Problems. London:

Routledge.

WITTGENSTEIN, L. (2005a). Philosophical Investigations. Oxford: Basil Blackwell.

WITTGENSTEIN, L. (1975). Philosophical Remarks. Oxford: Basil Blackwell. (2005). The Big Typescript. Oxford: Basil Blackwell. (1961). Tractatus Logico-Philosophicus. London: Routledge \& Kegan Paul.

.(1976). Wittgenstein's Lectures on the Foundations of Mathematics. (C. Diamond, Ed.) Hassocks: The Harvester.

WRIGHT, C. (1993). Realism, Meaning and Truth. Cambridge: Basil Blackwell. 